\title{
Recursos audiovisuales y educación
}

\section{Beatriz Susana Sevilla}

\section{Resumen}

El exponencial crecimiento de la información requiere políticas públicas para garantizar la accesibilidad universal a los recursos educativos en general y en particular a los recursos audiovisuales de calidad. Estrategias educativas para afrontar estos desafíos y para generar conocimientos brindando igualdad de oportunidades para todos. Hay ejemplos de Universidades españolas sobre la transferencia científica. Para garantizar la masiva producción y divulgación de recursos audiovisuales relevantes, pertinentes y actualizados, es importante desarrollar Recursos Educativos Abiertos y plataformas interinstitucionales, nacionales e internacionales.

\section{Palabras clave}

Recursos audiovisuales, educación, políticas de Estado, plataformas interinstitucionales, sistema único de catalogación, accesibilidad

\section{Audio-visual Resources and Education}

\section{Abstract}

The exponential information growth requires public policies to ensure the universal access to educational resources in general and in particular to quality audiovisual resources. Educational strategies to face these challenges and to generate knowledge to provide equal opportunities for all. There are some examples in Spain Universities about scientific transferce. To ensure the mass production and dissemination of relevant, pertinent and updated audiovisual resources, it's important to develop Open Educational Resources and interagency, national and international platforms.

\section{Keywords}

Audio-visual resources, education, State policies, inter-agency platforms, Multimedia literacy, unique cataloging system, accessibility

http://dx.doi.org/10.5209/rev_CDMU.2013.v24.46367 


\section{INTRODUCCIÓN}

Ante el exponencial crecimiento de la información generada por el ubicuo desarrollo científico y tecnológico se plantean serios interrogantes: ¿Qué medidas o políticas de Estado serían recomendables para garantizar la producción y la accesibilidad a la información en general y en particular a la referida a los recursos audiovisuales de calidad?¿Qué estrategias deben implementarse en educación para afrontar estos desafíos y para generar conocimientos brindando igualdad de oportunidades para todos ? ¿Cómo acceder a información relevante teniendo en cuenta creciente cantidad y su alto nivel de dispersión? ¿Qué rol pueden desempeñar los Recursos Educativos Abiertos y las plataformas interinstitucionales, nacionales e internacionales para garantizar una masiva producción y divulgación de recursos audiovisuales relevantes, pertinentes y actualizados?

Es habitual adjetivar a esta era como la de la sociedad del conocimiento o de la información, sin embargo sabemos que estos conceptos no son sinónimos. Es necesario destacar que la construcción del conocimiento implica diversos procesos por los cuales podemos apropiarnos de la información de modo consciente, más allá del mero acopio de la misma.

\section{REQUERIMIENTOS ACTUALES Y PERSPECTIVAS DE LA EDUCACIÓN}

Considerando el crecimiento exponencial de la tecnología y de la información científica, los sistemas educativos en general $y$, particularmente, el profesorado necesitan contar con herramientas que contribuyan a crear y a desarrollar entornos de aprendizaje centrados en el alumnado. Sin embargo los docentes suelen estar presionados por urgencias temporales y múltiples exigencias de innovación contrapuestas con la rigidez curricular. A esto se suman los requisitos de formación para crear sus propios materiales adaptados a su estilo de enseñanza, la diversidad cultural del alumnado, las limitaciones presupuestarias, la dispersión de múltiples entornos de aprendizaje y/o la existencia de diversos agentes educadores generalmente desconectados entre sí, entre otros factores.

\section{LA DE LOS RECURSOS AUDIOVISUALES EN LA ACTUALIDAD}

Son de indudable importancia las herramientas que aporta el movimiento web 2.0 para realizar trabajos colaborativos entre varios usuarios o colaboradores, no sólo para trabajar en diversos campos y producir información sino para mejorar y desarrollar nuevas actividades educativas tanto en el aula como fuera de ella. El desarrollo de la web 2.0 está provocando de forma indirecta una avalancha de contenidos audiovisuales: cada vez aparecen nuevas Web TV que difunden diversos contenidos y vídeos a través de las diferentes redes sociales como Facebook, Twitter, Youtube, Vimeo, entre otros.

Diversos géneros audiovisuales procedentes de otros entornos comunicativos están evolucionando para adaptarse a la lógica mediática de Internet y abren nuevas perspectivas para la creación de nuevos géneros narrativos exclusivos para la red y que pueden influir a su vez en otros ámbitos culturales.

Los efectos positivos de la difusión de estos recursos audiovisuales y del trabajo en red constituyen un aporte fundamental para promover una construcción colectiva del conocimiento, estimular la capacidad de relacionarse, discutir y argumentar. Son particularmente relevantes aquellos recursos audiovisuales producidos especialmente en centros de investigación científica y en universidades. Es deseable que su 
progresiva incorporación en diversos contextos pedagógicos potencien la interactividad entre los científicos, los integrantes del sistema educativo en sus diversos niveles y la comunidad en general.

Sin embargo la diversidad de plataformas y aplicaciones destinadas a difundir recursos educativos está en constante crecimiento y existe un alto grado de dispersión de la información, planteando muchas dificultades a los usuarios a la hora de realizar una búsqueda eficiente, confiable y ágil.

Considerando este escenario, surge entonces la necesidad de facilitar la accesibilidad no sólo a las comunidades científicas y docentes nacionales e internacionales de diversos niveles educativos sino a todos los usuarios potenciales de la sociedad que decidan emplearlos para su formación permanente.

La facilidad y rapidez de acceso a diversas fuentes de información no sólo estimularía al alumnado sino que proporcionaría a profesores e investigadores múltiples recursos y herramientas para integrar y enriquecer sus clases y proyectos.

\section{POLÍTICAS PÚBLICAS RECOMENDABLES PARA LA TRANSFERENCIA CIENTÍFICA Y TECNOLÓGICA MEDIANTE RECURSOS AUDIOVISUALES}

La iniciativa pública es o debería ser fundamental en cada país y a nivel internacional para garantizar la producción y la accesibilidad a la información en general, y en particular la referida a los recursos audiovisuales de calidad.

Se debería impulsar un cambio en el flujo informativo y comunicativo, orientado a la reorganización de los sistemas de producción y a la difusión de los contenidos audiovisuales generados por diversas instituciones de carácter público, para favorecer el acceso masivo y universal a los diversos contenidos digitales.

Sabemos que para concretar estas medidas es primordial contar con un claro interés de integración por parte de la mayoría de las instituciones así como con el trabajo efectivo y continuado de los equipos profesionales para concretar los cambios tecnológicos y operativos acordados.

Considerando el crecimiento exponencial del conocimiento, sería ideal contar con una plataforma universal de recursos audiovisuales y con un sistema único de catalogación organizados según pautas comunes (por ejemplo, las establecidas por UNESCO), preparada para incorporar meta-datos en forma progresiva, con capacidad de renovación permanente en función de nuevas producciones aportadas por distintos centros de investigación y producción. A medida que se sumen nuevas producciones al importante acervo ya existente, el flujo informativo debería renovarse constantemente para garantizar que el proceso de actualización del conocimiento en las diversas disciplinas científicas sea divulgado masivamente.

El proceso que, con optimismo, podría llevar a concretar un ambicioso emprendimiento de estas características, tendría que ir acompañado indefectiblemente de una masiva divulgación orientada a los diversos centros de investigación, a todos los niveles y sistemas educativos y a los usuarios en general, a través de los diversos soportes tecnológicos vigentes y futuros: canales de televisión (nacionales, universitarios, de las Comunidades Autónomas), redes sociales, dispositivos móviles, etc. 


\section{INICIATIVAS EN ESPAÑA}

Un ejemplo de apertura de los centros universitarios a la comunidad lo constituye el proyecto de los responsables de los Servicios Audiovisuales de las Universidades Españolas (SAVUES) que han comenzado a trabajar desde 2012 en el diseño y desarrollo de una plataforma compartida, para integrar el conjunto de los repositorios de contenidos audiovisuales universitarios y para ordenarlos y seleccionarlos de manera accesible para los usuarios. Con esto pretenden facilitar las búsquedas en el nuevo flujo semántico e interactivo de la red y favorecer una mayor apertura a la sociedad para comunicar mejor el conocimiento y la investigación.

http://www.ub.edu/web/ub/es/menu eines/noticies/2012/10/020.html II Jornadas de los Servicios Audiovisuales de las Universidades Españolas

Otra iniciativa concreta surgida desde el campo universitario español es el proyecto "La Universidad responde" realizado por los servicios de audiovisuales de las 10 universidades participantes,a partir de un convenio de colaboración para diseñar, realizar,compartir y difundir esta serie integrada por cerca de un centenar de vídeos de dos minutos de duración. Mediante un lenguaje cercano y comprensible para el gran público, se pretende dar a conocer aquellos descubrimientos y adelantos científicos que pueden contribuir a mejorar la calidad de vida y a facilitar las actividades cotidianas de las personas. Integran este proyecto: la Universitat d'Alacant, la Universitat Autònoma de Barcelona, la Universitat de Barcelona, la Universidad de Educación a Distancia, la Universitat de les Illes Balears, la Universidad de Salamanca, la Universidad de Santiago de Compostela, la Universidad Politécnica de Valencia, la Universitat de València y la Universidade de Vigo.

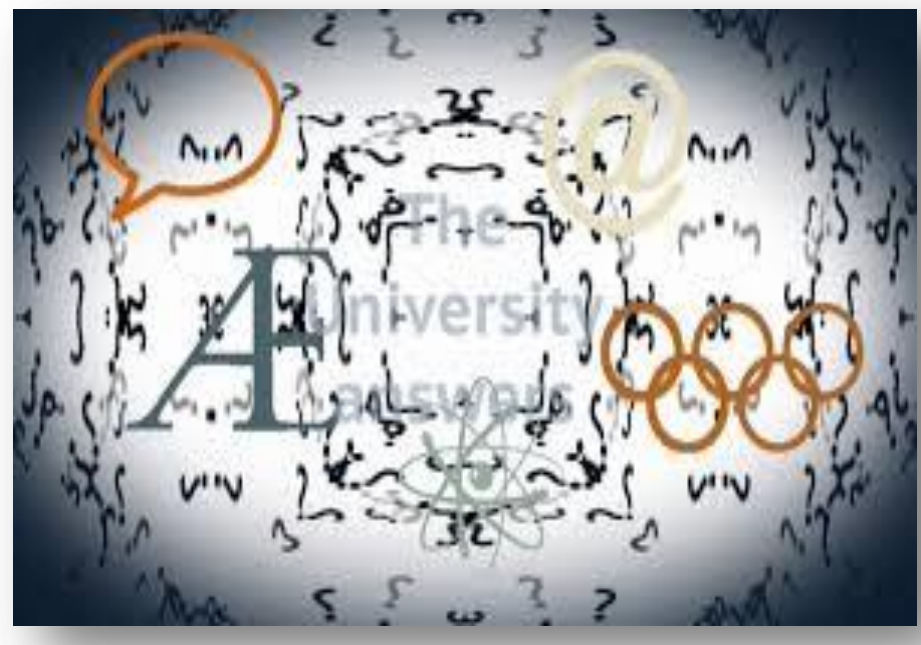

Micro espacios donde expertos dan a conocer diversos conocimientos

La CRUE presenta la serie "La universidad responde" https://www.uib.es/es/noticies/Canal-video/-CRUEpresenta-a-la-prensa-la-serie-La-Universidad.cid291990 
Este tipo de proyectos debería mulplicarse considerando la gran cantidad y variedad de recursos audiovisuales con contenidos significativos y de relevancia científica, social y cultural, que actualmente se encuentran dispersos en distintas páginas Web y que están catalogadas con diversidad de criterios.

Son de particular interés aquellas producciones audiovisuales documentales con contenidos científicos, qur difunden los avances en la investigación y el desarrollo tecnológico en diversas disciplinas y proyectos multidisciplinarios, que producen y /o divulgan las instituciones estatales a través de sus páginas Web, como por ejemplo el Consejo Superior de Investigaciones Científicas, RTVE, centros de investigación científica como CEMAV- ASECIC., las Universidades, etc.

Es recomendable profundizar las estrategias de integración interinstitucional para superar la dispersión y la desinformación. Con la esperanza de que esto pueda concretarse y para demostrar sintéticamente esta realidad, adjunto un Anexo con una serie de enlaces a las instituciones españolas e iberoamericanas que aportan recursos audiovisuales educativos y de divulgación científica.

Esto requiere sin duda otro tipo de estrategias complementarias e indispensables para garantizar el acceso personal y social a la información, como son las referidas a la alfabetización multimedia.

\section{LAS ESTRATEGIAS DE ALFABETIZACIÓN MULTIMEDIA}

Es fundamental incentivar la capacidad crítica en relación con las fuentes de información en general y con respecto a los materiales didácticos en particular, entre los cuales se incluyen los recursos audiovisuales con contenidos de relevancia científica, social y cultural.

Entre los objetivos de la educación se destaca el desarrollo de la capacidad de búsqueda de información con criterios sólidos, indagando sobre las fuentes (su origen y autoría), para poder considerarlas confiables y, en lo posible, procurar contrastar diversos puntos de vista o enfoques acerca del tratamiento de una temática determinada, comprender su evolución y estar permanentemente actualizados sobre los avances o descubrimientos en la materia.

Es elemental para los usuarios conocer no sólo las características y conceptos básicos de catalogación, sino también también es sustancial verificar la procedencia institucional y la autoría de cada una de las producciones audiovisuales, la calidad de la información sobre los contenidos fundamentales, valorando si, además, aporta recursos adicionales que permitan ampliar, complementar o profundizar sobre su temática, tanto para investigar sobre la misma (textos en diversos formatos, imágenes, bibliografía, webgrafía, etc.) como emplearlas como recurso en educación (guías didácticas y de auto-aprendizaje, referencia al nivel educativo recomendado, etc.). Otros datos a tener en cuenta son las fechas de edición del audiovisual así como la información sobre cuándo se produjeron u originaron sus contenidos.

Los recursos audiovisuales proporcionarán valor agregado a la construcción social del conocimiento si además incluyen una opción participativa que permita a los usuarios exponer, compartir e intercambiar comentarios y/o relatos sobre sus experiencias relacionadas con el uso (científico, educativo, laboral, social) de dichos recursos. 
Teniendo en cuenta la opinión de los beneficiarios, la búsqueda puede orientarse en función de aquellos vídeos más valorados por el público en general, por las comunidades científicas y por los integrantes de distintos niveles educativos. La pluralidad de enfoques contribuirá a ofrecer a los especialistas y a la sociedad en general una visión amplia acerca de las diversas disciplinas científicas y sobre su aplicación, evolución e interconexión.

Cada persona debería ser capaz de filtrar, contextualizar, ordenar y seleccionar recursos audiovisuales para mejorar el rendimiento académico o laboral, enriquecer las prácticas innovadoras en educación y, fundamentalmente, para desarrollar su educación en forma permanente, a lo largo de toda la vida.

Los portadores de esta alfabetización multimedia estarían capacitados para emplear todo tipo de recursos audiovisuales con fines educativos, sociales y culturales, especialmente teniendo en cuenta la creciente producción de REA (Recursos Educativos Abiertos) en formatos digitales de normas abiertas, así como el extendido uso de las redes sociales.

Está comprobado el valor motivador y educativo que tienen los recursos audiovisuales para la infancia y la adolescencia. Conocer cómo se producen y aprender a realizarlos les permite mayor capacidad crítica sobre los mismos y les brinda herramientas para expresarse y comunicar sus experiencias de aprendizaje y de todo tipo. Por ello es importante respaldar a las diversas instituciones que apuestan por formar y motivar a profesores y demás integrantes de la comunidad educativa para que produzcan e intercambien materiales educativos accesibles y de alta calidad, incluyendo los audiovisuales teniendo en cuenta las necesidades locales y la diversidad de los estudiantes.

Este tipo de actividades de producción reúne las características sinérgicas del trabajo en equipo, el desarrollo de habilidades adecuadas a los distintos tipos de inteligencias múltiples (Howard Gardner), la convergencia de diversas disciplinas y asignaturas para desarrollar proyectos centrados en el aprendizaje del alumnado con la guía del profesorado, fortaleciendo nuevos enfoques curriculares como la enseñanza bimodal o de clase invertida (flipper classroom).

Estas experiencias participativas basadas en los intereses e inquietudes del alumnado permiten desarrollar actitudes solidarias entre ellos y con su contexto, y además generan el desafío de registrarlas con diversos soportes y lenguajes, para difundirlas como nuevos tipos de recursos de enseñanza-aprendizaje. Estas nuevas formas de producción social del conocimiento deberán tenerse en cuenta a la hora de sistematizar y diseñar plataformas con fines educativos, considerando además la creación de mecanismos para su evaluación y para la certificación de los resultados para difundirlos como Recursos Educativos Abiertos (REA).

\section{GENERAR Y DIFUNDIR INFORMACIÓN CON LOS REA}

Es fundamental procurar difundir y utilizar los recursos, repositorios o plataformas que contienen valiosa información y recursos educativos abiertos en forma más estratégica, porque lo que realmente es escaso es el tiempo y la capacidad de atención. La creciente información de valor no siempre puede ser plenamente utilizada porque se desconoce dónde o cómo buscarla. 
Por ello valoramos (especialmente desde un contexto de crisis) las recomendaciones de la UNESCO consignadas en la Declaración de París de 2012 sobre Recursos Educativos Abiertos (REA), para que los Estados inviertan con fondos públicos en la producción y el uso de recursos educativos abiertos en idiomas locales para que los mismos estén disponibles con licencias abiertas y tengan mayor impacto en la calidad de vida de la población.

Es primordial elaborar, compartir y utilizar los REA en formatos digitales de normas abiertas para ampliar el acceso y mejorar la calidad de la enseñanza-aprendizaje formal y no formal y en diversos contextos culturales, en aras de su pertinencia y accesibilidad.

En síntesis: para garantizar la producción y la accesibilidad universal a los recursos educativos en general y en particular a los recursos audiovisuales de calidad se requieren tanto políticas públicas o de Estado que promuevan la producción y distribución de la información científico-técnica a través de Recursos Educativos Abiertos y plataformas interinstitucionales nacionales e internacionales.

Para lograr procesos de enseñanza aprendizaje significativos y eficaces, tanto en entornos presenciales como virtuales, es fundamental brindar a todos la oportunidad de acceder a recursos audiovisuales motivadores y de auto-aprendizaje relevantes, pertinentes y actualizados que contribuyan a enriquecer los demás recursos educativos, atendiendo a las necesidades de una gran diversidad de usuarios.

El verdadero desafío es no sólo generar información de valor sino lograr su distribución universal.

\section{REFERENCIAS:}

Declaración de París de 2012 sobre los REA (recursos Educativos Abiertos)

http://www.unesco.org/new/fileadmin/MULTIMEDIA/HQ/CI/CI/pdf/Events/Spanish Paris OER Decl aration.pdf

Presentación de las Directrices sobre recursos educativos abiertos de la UNESCO y el Commonwealth of Learning en la Conferencia General de la UNESCO_http://www.unesco.org/new/es/mediaservices/single-view-tv-

release/news/unescocommonwealth of learning oer policy guidelines to be launched at the unesco general conference/

Que es el aprendizaje invisible, por Cristóbal Cobo Romaní

http://ccollection.unia.es/videos/qu\%C3\%A9-es-el-aprendizaje-invisible-entrevista-crist\%C3\%B3balcobo

Portal de recursos educativos abiertos http://www.temoa.info/es

CLARISE "Comunidad Latinoamericana Abierta Regional de Investigación Social y Educativa”http://sites.google.com/site/redclarise/

Donde encontrar REA? https://sites.google.com/site/redclarise/rea 
Wikimedia Commons http://commons.wikimedia.org

Los servicios audiovisuales universitarios apuestan por crear una plataforma única de contenidos http://www.unia.es/content/view/2065/165/

http://www.rtve.es/alacarta/videos/la-aventura-del-saber/aventura-del-saber-howardgardner/1856341/ Howard Gardner, teoría de las inteligencias múltiples

https://www.youtube.com/watch?v=l2QtSbP4FRg Howard Gardner, teoría de las inteligencias múltiples (en inglés)

\section{ANEXO I}

\section{Instituciones públicas con recursos audiovisuales en España}

1. Servicio de Información y Noticias Científicas (SINC) http://www.agenciasinc.es/ Es la primera agencia pública de ámbito estatal especializada en información sobre ciencia, tecnología e innovación en español. El equipo de SINC produce noticias, reportajes, entrevistas y materiales audiovisuales (vídeos, fotografías, ilustraciones e infografías). Todos los contenidos producidos por SINC tienen una licencia Creative Commons 2.5,

2. Plataforma SINC (del FECYT). SINC, la ciencia es noticia

3. Cienciatk Plataforma del Consejo Superior de Investigaciones Científicas (CSI) http://www.cienciatk.csic.es/que es cienciatk/ Pretende divulgar y acercar a los ciudadanos la actividad científica y técnica. Ofrece la posibilidad de visionar y compartir a través de Internet, vídeos documentales, fotografías y sonidos de carácter científico técnico.

4. Archivo audiovisual de Radio Televisión Española RTVE cuenta con un enorme archivo audiovisual de documentales y programas de gran interés educativo y cultural, accesible a todo público. http://www.rtve.es/archivo/ http://www.rtve.es/alacarta/

http://www.rtve.es/television/canal-cultural/otras-emisiones/

5. Videoteca de la Consejería de Educación de la Comunidad de Madrid. Mediateca Educa Madrid http://mediateca.educa.madrid.org/

6. Web arte-historia Junta de Castilla y León http://www.artehistoria.jcyl.es/

Vídeos con textos, interesantes recursos para historia y el arte, ejemplos: http://www.artehistoria.jcyl.es/v2/videos/

Canal Arte Historia Youtube

https://es.video.search.yahoo.com/search/video; ylt=A9mSs2r.sMtTDDQA7..T.Qt.; ylu=X3o DMTBsYWhiN2NvBHNIYwNzYwRjb2xvA2lyMgR2dGlkAw--?p=canal+arte+historia 
7. Plataforma Audiovisual ASECIC (Asociación Española de Cine e Imagen Científicos) http://asecic.org/

http://asecic.org/plataforma-audiovisual/documentales/canales-asecic

Canales ASECIC Contenidos audiovisuales producidos por miembros de la Asociación Española de Cine e Imagen Científicos

Contenidos audiovisuales distribuidos en otros canales o web tv http://asecic.org/plataforma-audiovisual/documentales/otros-canales

8. Centro de Astrobiología asociada a la NASA Astrobiology Institutye

http://cab.inta-csic.es/es/inicio multimedia: fotos y vídeos

http://cab.inta-csic.es/es/multimedia/videos Videos

9. Cervantes virtual. Videoteca http://bib.cervantesvirtual.com/videoteca/

Cervantes TV es la televisión por Internet del Instituto Cervantes- Difunde la actividad cultural y académica en español que se celebra en el mundo http://tv.cervantes.es/sobre-cervantes-tv/El canal 3 de Cervantes TV recoge todos los vídeos realizados o difundidos por el Instituto Cervantes en colaboración con otras instituciones.

10. Edu3.cat Portal educativo del Departamento de Educación de la Generalitat de Cataluña y la Corporación Catalana de Medios Audiovisuales. http://www.edu3.cat/Edu3tv/Cerca?p tip=\&p ftr=2\&p cp=1\&p amb=\&p niv=\&p are=\&p ex $=\& p$ to $=\& p$ alg $=\& x=19 \& y=17$ (en catalán)

11. CANAL SUR 2 Programa TESIS http://www.cedecom.es/tv/tesis.html Contenidos audiovisuales emitidos en el programa TESIS de CANAL SUR 2 ANDALUCÍA. Es un programa de televisión diferente pionero de todos los existentes... El programa Tesis, ha sido creado y producido por Cedecom,

\section{Portales de vídeos y TV de las Universidades de España}

1. UBtv La televisión de la Universidad de Barcelona http://www.ub.edu/ubtv/

https://www.youtube.com/user/UB

Servei d' Audiovisuals http://www.ub.edu/audiovisuals/laudiovisual-al-servei-de-launiversitat

2. Vídeos y TV educativa Universidad Nacional de Educación a Distancia http://portal.uned.es/portal/page? pageid=93,1126889\& dad=portal\& schema=PORTAL 
https://canal.uned.es/

Vídeos y programas de TV de UNED en Youtube https://www.youtube.com/user/uned/videos?flow=grid\&view $=0$

Canal CEMAV- ASECIC http://www.uned.es/cemav/asecic/ El objetivo de este canal es presentar y ofrecer una muestra audiovisual permanente de las distintas producciones que realizan tanto el Centro de Medios Audiovisuales (CEMAV) de la UNED, como las entidades miembros de la ASECIC.

3. Universidad Autónoma de Madrid Geoteca

http://vimeo.com/geoteca/videos todos los vídeos que GEOteca video ha subido a Vimeo. Las apariciones son vídeos en que otras personas le han dado crédito a GEOteca video.

4. Canal UPCTV Videoteca de la Universidad Politécnica de Cataluña. http://tv.upc.edu/?set language=es

5. Canal UVic Videoteca de la Universidad de Vic catalán) https://www.youtube.com/user/UniversitatdeVic

6. Deusto TV TV de la Universidad de Deusto http://www.deusto.tv/blog/?page id=2 http://www.youtube.com/user/deusto/videos?view=0

7. UIB Universitat de les Illes Baleares

Canales temáticos:http://canal.uib.cat/es/canals/

Incluye: académicos, divulgación e investigación, campus, series, animación.

8. Canales de radio y TV de la Universidad Politécnica http://www.upv.es/pls/oreg/rtv web.TVCarta?p_idioma=C archivo de vídeos de conferencias y entrevistas

9. Universidad de Valencia

https://www.youtube.com/user/UVMEDIA

Producciones de vídeo del Taller de Audiovisuales de la Universidad de Valencia. http://www.uv.es/legalskills/legalskills/Videoteca.html

MediaUni Televisión IP de la Universidad de Valencia http://mediauni.uv.es/ Videoteca no muy completa, es más bien institucional

10. Área de Audiovisuales de la Universidad Carlos III de Madrid

https://arcamm.uc3m.es/arcamm/ 


\section{UC3M-TV}

Clases por televisión con imágenes; ejemplo: http://marge2.uc3m.es/arcamm/index.php\# Sociología del género en España

11. Canal Audiovisual de la Universidad Internacional de Andalucía

$\underline{\text { UNIAtV }}$ http://blogs.unia.es/uniatv/ (archivo de vídeos de conferencias y entrevistas)

$\underline{\text { http://blogs.unia.es/uniatv/archives }}$

12. Televisión online de la Universidad de Sevilla http://tv.us.es/

Videoteca Universidad de Sevilla

Portal de videos de la Universidad de Sevilla (hay muchísimos vídeos institucionales, cuesta encontrar otros educativos, pero los hay...) Ejemplo: http://tv.us.es/la-camara-oscura-entre-la-magia-y-la-ciencia/

13. TV de la Universidad de Santiago de Compostela USC TV USC TV - MEDIATECA (en gallego y castellano) Recursos educativos http://tv.usc.es/educa

14. Vídeos de la Universidad Autónoma de Barcelona Vídeos de la UAB la mayoría son vídeos institucionales y también hay vídeos de divulgación científica (en catalán y castellano) Ejemplo: Sinfonía celular http://www.youtube.com/watch?v=Z_Hk3XD8k w\&feature=relmfu

\section{Instituciones internacionales con recursos audiovisuales}

1. UNESCO TV (ESPAÑOL) http://www.youtube.com/unescospanish Ejemplos uso de vídeos documentales en educación

Para historia antigua: Cartago en 3D

http://www.youtube.com/watch?v=9lqk4UXthjc\&list=UUvZQCv0TqDHVtdOg5X5OnIA\&index $=108 \&$ feature $=$ plcp

2. UNICEF Comité Español http://www.youtube.com/unicefESP

Ejemplos: $\quad$ Enrédate con Unicef http://www.youtube.com/playlist?list=PLC461D0D77F783336\&feature=plcp

3. Vídeos de la Agencia Espacial Europea (ESA). http://www.spacetelescope.org/videos/viewall/ (en inglés)

ESO, el Observatorio Europeo Austral, http://www.eso.org/public/spain/ construye y opera un conjunto de telescopios astronómicos basados en tierra de los más avanzados del mundo. Archivo completo de vídeos (en inglés)

4. MIT Tech TV Videoteca del Massachusetts Institute of Technology. http://techtv.mit.edu/ (en inglés) 
Ejemplo, arte: música http://techtv.mit.edu/collections/arts/videos/18196-awakening-mitwind-ensemble-rehearsal

5. Vídeos de la NASA http://www.nas.nasa.gov/publications/gallery.html Fondo videográfico de la Agencia Estadounidense del Espacio y la Aeronáutica.

6. $\mathrm{NCl}$ ATEI Noticias Culturales Iberoamericanas. http://www.nci.tv/

Videoteca con girtual guías didácticas

http://www.nci.tv/index.php/menueducacion/subsubmenu-videoteca/subsubsubmenu-

guias-didacticas

7. Khan Academy http://www.khanacademy.org/ (en ingles) recursos para el autoaprendizaje personalizado https://es.khanacademy.org/ en español: visionado, ejercicios prácticos, coach, voluntarios. Los profesores, padres o entrenadores pueden comprobar el rendimiento de sus alumnos.

http://www.youtube.com/user/KhanAcademyEspanol misión: acelerar el aprendizaje de estudiantes de todas las edades.

8. Arkive org http://www.arkive.org/about/ (en inglés) promueve la conservación de especies amenazadas en el mundo a través del poder de las imágenes de la vida silvestre. (Films y fotografías)De libre acceso para todos. Interesante el apartado con las universidades varios países.

9. Videoteca virtual de la FCA Facultad de Contaduría y Administración de la UNAM. http://videoteca.fca.unam.mx/videoteca/index.php\#contenido

10. Learning zone broadband Class clips http://www.bbc.co.uk/learningzone/clips/ Clase Clips es un sitio de más de 10.000 clips de vídeo y audio, con fines educativos. BBC series y otros.

11. RAI Radio y televisión italiana, rai.escuela

http://www.raiscuola.rai.it/ http://www.raiscuola.rai.it/lessonPlan.aspx

12. tvfrance5

http://www.france5.fr/

http://documentaires.france5.fr/index.php

13. TV Educa : La Televisión Educativa por Internet http://www.tveduca.com/ Red Educativa Mundial REDEM:plataforma de difusión y manejo de nuevas herramientas, metodologías de enseñanza,experiencias compartidas por docentes, centros e instituciones educativas a nivel internacional en sus diferentes formas y niveles de educativos.

14. Televisión educativa de México http://televisioneducativa.gob.mx La Dirección General de Televisión Educativa (DGTVE), difunde material audiovisual educativo, cultural y complementario que sirve como apoyo para profesores, alumnos y público en general. Videoteca Educativa de las Américas (VELA) http://vela.sep.gob.mx/

15. Argentina: Canal encuentro http://www.encuentro.gov.ar/

16. Chile: Canal NOVASUR http://www.novasur.cl/ 


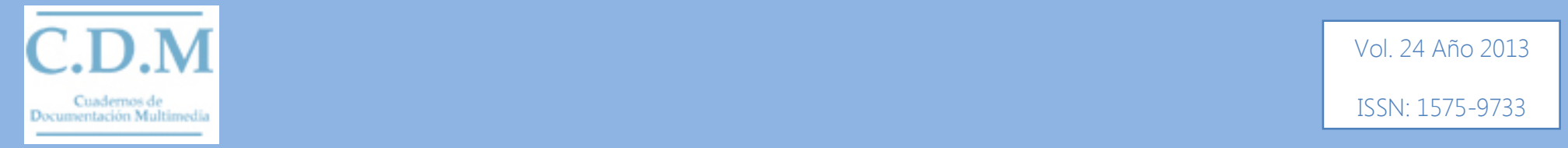

17. Red Universitaria Nacional de Chile REUNA www.reuna.cl 